\title{
EDITORIAL
}

\section{The pressure mounts on lung dendritic cells}

\author{
B.N. Lambrecht and L.M. van den Toorn
}

$\Delta$ role for inflammatory processes in the pathogenesis of pulmonary arterial hypertension (PAH) has been proposed, since inflammatory infiltrates surrounding remodelled vessels in human and experimental PAH have been described by several groups [1, 2]. Increased levels and expression of cytokines, chemokines and lipid mediators have been found in the blood, lung parenchyma and affected pulmonary vessels. The higher prevalence of $\mathrm{PAH}$ in connective tissue disorders and the successful treatment with immunosuppressive therapy in some forms of PAH support the concept of inflammatory pathways in the development and/or modulation of the disease.

Although data are scarce, the infiltrates found in plexiform lesions consist mainly of lymphocytes and macrophages, whereas neutrophilic inflammation has not traditionally been described. The presence of lymphocytes in the vessel wall suggests that these cells might control the disease process by secretion of pleiotropic cytokines, analogous to what has been described in other T-cell-mediated diseases, such as asthma, auto-immune disorders or transplant rejection. In these diseases, effector T-lymphocytes are activated locally by antigen-presenting dendritic cells (DCs) to mediate disease $[3,4]$. A report by Perros et al. [5] in the current issue of the European Respiratory Journal describes an increase in these DCs in plexiform lesions of idiopathic PAH (IPAH) patients and rats with monocrotaline-induced $\mathrm{PAH}$. This strongly suggests that these DCs present some form of (auto-)antigen to T-cells that might initiate or perpetuate inflammation.

DCs are professional antigen-presenting cells that are located throughout the body but particularly at the boundaries of the body, such as mucosal surfaces and the skin, where they are known as Langerhans' cells. In these peripheral sites, they are specialised in recognising foreign antigens that invade across the natural barrier of the skin and mucosa [3,4]. At this stage, they are in a so-called immature state because they do not yet present antigen to T-lymphocytes. Upon recognition of foreign antigen, they migrate via the local afferent lymphatics to the draining lymph nodes where naïve $\mathrm{T}$ - and B-lymphocytes are continuously recirculating. By the time they arrive in the lymph node, they have processed the antigen into small peptides for presentation onto major histocompatibility complex class I and class II molecules, and they now express an

Dept of Pulmonary Medicine, Erasmus University Medical Center, Rotterdam, The Netherlands.

STATEMENT OF INTEREST: None declared.

CORRESPONDENCE: B.N. Lambrecht, Dept of Pulmonary Medicine, Room Ee2251, Erasmus MC Dr Molewaterplein 50, 3015GE Rotterdam, The Netherlands. Fax: 31 104089453. E-mail: b.lambrecht@erasmusmc.nl array of so-called co-stimulatory molecules (CD80, CD86, CD83) that are required for activation of naïve T-cells. The attraction of immature DCs to the periphery of the lung and the process of functional maturation is induced mainly by recognition of "foreign" dangerous antigens that contain pathogen-associated microbial patterns, such as Toll-like receptor ligands (endotoxin, peptidoglycan, viral and bacterial RNA or DNA) [6], but can also be induced by damageassociated molecular patterns (DAMPs), which are released by stressed tissues and by necrotic cell death, such as uric acid, heat shock proteins and high mobility group box 1 protein $[7$, 8]. Mature DCs determine the outcome of adaptive immunity in the draining nodes and they instruct a tailor-made immune response by T-cells (T-helper cell (Th) type 1, Th2 or Th17, depending on the type of pathogen) and B-cells [9]. DCs are also crucial for immune homeostasis as, in the absence of a sense of danger to a particular (self-)antigen, they mediate tolerance by induction of so-called regulatory T-cells (Tregs), which suppress inflammation and protect from auto-immune damage [10, 11]. Activated DCs that present auto-antigen can induce auto-immune disease in a number of disease models, in which case interleukin (IL)-17-producing Th cells (Th17) are predominantly found.

The paper by PERros et al. [5] shows that DCs are found in the vessel wall of patients with IPAH and rats with experimentally induced PAH. This is striking, as the pulmonary arteries are not frequently exposed to foreign pathogens. The most likely explanation for the increased numbers of DCs in this region would be the recruitment of DCs in response to tissue damage and the release of the associated DAMPs. It is indeed known that oxidative damage occurs inside the vessel wall of plexiform lesions, and oxidative damage is by itself an activator of the DC system [12]. Exactly how DCs end up in the vessel wall is not mentioned in the article by PERROS et al. [5], but several candidate mechanisms might come into play. Peripherally based immature DCs that are recruited into inflammatory foci can derive from a monocytic precursor that circulates in the blood [13]. The mechanisms governing monocyte egress to the periphery are slowly being elucidated but clearly the chemokine fractalkine $\left(\mathrm{CX}_{3} \mathrm{CL}\right)$ is crucial in this process, as DC precursors express the fractalkine receptor. Also, the chemokine RANTES (regulated on activation, normal T-cell expressed and secreted) is involved in recruiting immature DCs (precursors) to the periphery. Strikingly, BALABANIAN et al. [1] and DORFMÜLLER et al. [2] have previously shown that these chemokines are expressed in high levels in plexiform lesions and serum of IPAH patients. An alternative explanation for the accumulation of DCs in the vessel wall might be a defect in their migration to the draining lymph nodes. One area that certainly requires further investigation is 
the role that the lipid mediator platelet-activating factor (PAF) might play. Particularly in the monocrotaline-induced rat model of PAH, the levels of PAF are greatly increased and a PAF antagonist prevents PAH in this model [14]. It has recently been shown that PAF immobilises DCs in the periphery and is responsible for the accumulation of DCs in the skin and vessel wall of dyslipidaemic ApoE-/- mice, where DCs initiate atherosclerotic plaques [15]. The hypothesis that PAF is required for DC accumulation in the rat model of PAH could be tested due to the availability of PAF antagonists.

The most important question that arises from the studies of PERros et al. [5] and DORFMÜLLER et al. [2] is what the functional role of DCs might be in PAH. The localisation of lymphocytes and DCs in one site suggests that these cells are functionally communicating [4]. For this assumption to gain acceptance, it would have been more complete if the authors had shown that DCs clustered with T-cells inside the vessel wall. The functional proof for a role for DCs in mediating PAH would require an animal model where DCs can be conditionally depleted. Such models are available in the mouse [16] but not in the rat, and could prove invaluable in proving that DCs do indeed mediate a crucial process in the pathogenesis of $\mathrm{PAH}$. However, mice are more resistant to the pulmonary vascular effects of monocrotaline.

If DCs do contribute to pathogenesis, the next question that arises is what (auto-)antigen they might be presenting. Antibodies to endothelial cells, fibroblasts and nuclear antigens are found in the serum of patients with IPAH and collagen vascular disease-associated PAH [17-19]. The fact that $\mathrm{PAH}$ also occurs in several auto-immune diseases suggests that auto-antigens might trigger or maintain the process underlining vascular remodelling [17]. One striking observation was that the DCs found in the lesions were of immature phenotype, i.e. not expressing CD83 as a maturation marker. Normally, DCs that are found in inflammatory lesions contacting T-cells are mature and restimulate T-cells to mediate disease [20]. The reasons for the immature state of DCs inside the vessel wall of PAH lesions are obscure but could involve the increased expression of vascular endothelial growth factor (VEGF) inside these lesions. VEGF is thought to be responsible for the endothelial proliferation and misguided angiogenesis typical of plexiform lesions but is also known as a DC-suppressive cytokine [21]. Particularly in the cancer field, VEGF is described as a factor that keeps DCs in an immature state inside tumour lesions, thus preventing the induction of anti-tumoural immunity. Similarly, the activity of transforming growth factor (TGF)- $\beta$ isoforms is a hallmark of IPAH lesions. TGF- $\beta$ can suppress the maturation and function of DCs [22]. Finally, as no information is available on how the patients described in the study were treated prior to lung transplantation, it could also be that various treatment modalities altered the maturation state of the DCs (see later).

If DCs are recruited to the typical lesions of IPAH, could targeting their function modify disease progression? As of yet, the answer can only be speculative. If an auto-immune component plays a role in disease maintenance or progression, then removing DCs could be beneficial [17]. Alternatively, DCs might be present inside the lesions to suppress disease progression. This is certainly a possibility, in view of the immature state of the DCs. Generally speaking, immature DCs presenting (auto-)antigen contribute to immune homeostasis by induction of Treg cells and, thus, the presence of DCs might be a way of suppressing immune damage [23, 24]. Until specific DC depletion experiments can be performed in an animal model, an answer to this question will not be easily obtained.

Upon reviewing the currently available medical therapies for $\mathrm{PAH}$, the present authors were struck by how many of the current and future proposed drugs already target DC function. Systemic or inhaled prostacyclin analogues are leading agents for the treatment of severe $\mathrm{PAH}$, and the present authors suspect that many patients were receiving this therapy prior to lung transplantation. The present authors and others have recently shown that inhaled iloprost strongly modifies the function of lung DCs and suppresses their potential to induce effector T-cell responses $[25,26]$. Iloprost also suppresses the potential of DCs to migrate to the mediastinal nodes and therefore could be responsible for retaining DCs inside blood vessels. Strikingly, iloprost, cicaprost and treprostinil also reduce the production of pro-inflammatory cytokines and chemokines in DCs, while inducing the immunoregulatory cytokine IL-10 [26]. Furthermore, combined endothelin (ET) receptor $\mathrm{A}$ and $\mathrm{B}$ antagonists (ERAs, e.g. bosentan) also alter the functional properties of DCs [27]. The biological effects of ET-1 are not limited to its potent vasoconstricting activity. DCs produce large amounts of ET-1 and significantly increase the expression of endothelin receptors upon maturation. Selective blockade of the ET-A receptor significantly reduced expression of the mature DC marker CD83, decreased the production of the immunostimulatory cytokine IL-12, downregulated DC ability to stimulate T-cells, and promoted DC apoptosis. Conversely, selective ET-B receptor blockade resulted in increased expression of CD83 and improved DC survival. Therefore, ET-1/ET-A/ET-B autocrine/paracrine loops on DCs appear to be essential for the normal maturation and function of human DCs [27]. How this modifies disease progression in $\mathrm{PAH}$ patients taking these drugs is unclear at present. ERAs with specificity for the endothelin-receptor subtype A, including sitaxsentan and ambrisentan, are currently undergoing investigation in $\mathrm{PAH}$ and might more specifically target DCs.

In recent years, the phosphodiesterase type 5 inhibitor sildenafil has been used to treat PAH. Sildenafil has profound effects on monocyte differentiation and restores the immunosuppressive milieu in tumours by altering antigen-presenting cell function [28]. As stated by the PerRos et al. [5], the tyrosine

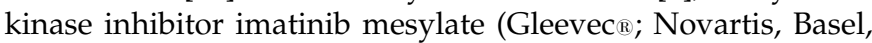
Switzerland, which inhibits c-kit, Abl and platelet-derived growth factor receptor- $\alpha$ ) has been proposed as a novel form of treatment for PAH. This compound induces a novel subset of DCs that has both natural killer and DC characteristics [29]. Whether this would contribute to the clinical effects of this compound in PAH remains an interesting hypothesis. First, a more detailed phenotypic analysis of DCs in PAH lesions will have to be performed. The lack of significant effects of systemic steroids in IPAH patients provides an argument against the role of DCs in PAH. DCs are exquisitely sensitive to the effects of steroids; however, it could be that steroids are given too late in disease pathogenesis. 
In conclusion, the article by PERROS et al. [5] opens up a new avenue of research in the dendritic cell and pulmonary arterial hypertension field that will hopefully clarify some basic aspects on the pathogenesis and treatment of this devastating disease. The pressure is now on.

\section{REFERENCES}

1 Balabanian K, Foussat A, Dorfmuller $\mathrm{P}$, et al. CX(3)C chemokine fractalkine in pulmonary arterial hypertension. Am J Respir Crit Care Med 2002; 165: 1419-1425.

2 Dorfmüller P, Zarka V, Durand-Gasselin I, et al. Chemokine RANTES in severe pulmonary arterial hypertension. Am J Respir Crit Care Med 2002; 165: 534-539.

3 Banchereau J, Steinman RM. Dendritic cells and the control of immunity. Nature 1998; 392: 245-252.

4 Lambrecht BN, Hammad H. Taking our breath away: dendritic cells in the pathogenesis of asthma. Nat Rev Immunol 2003; 3: 994-1003.

5 Perros F, Dorfmuller P, Souza R, et al. Dendritic cell recruitment in lesions of human and experimental pulmonary hypertension. Eur Respir J 2007; 29: 462-468.

6 Kadowaki N, Ho S, Antonenko S, et al. Subsets of human dendritic cell precursors express different toll-like receptors and respond to different microbial antigens. J Exp Med 2001; 194: 863-869.

$7 \mathrm{Gu}$ Y, Hershfield MS, Cohen A. The danger within. N Engl J Med 2004; 350: 2721-2722.

8 Skoberne M, Beignon AS, Bhardwaj N. Danger signals: a time and space continuum. Trends Mol Med 2004; 10: 251257.

9 Lambrecht BN, Prins JB, Hoogsteden HC. Lung dendritic cells and host immunity to infection. Eur Respir J 2001; 18: 692-704.

10 De Heer HJ, Hammad H, Soullie T, et al. Essential role of lung plasmacytoid dendritic cells in preventing asthmatic reactions to harmless inhaled antigen. J Exp Med 2004; 200: 89-98.

11 de Heer HJ, Hammad H, Kool M, Lambrecht BN. Dendritic cell subsets and immune regulation in the lung. Semin Immunol 2005; 17: 295-303.

12 Kantengwa S, Jornot L, Devenoges C, Nicod LP. Superoxide anions induce the maturation of human dendritic cells. Am J Respir Crit Care Med 2003; 167: 431437.

13 Geissmann F, Jung S, Littman DR. Blood monocytes consist of two principal subsets with distinct migratory properties. Immunity 2003; 19: 71-82.

14 Ono S, Voelkel NF. PAF antagonists inhibit monocrotalineinduced lung injury and pulmonary hypertension. J Appl Physiol 1991; 71: 2483-2492.
15 Angeli V, Llodra J, Rong JX, et al. Dyslipidemia associated with atherosclerotic disease systemically alters dendritic cell mobilization. Immunity 2004; 21: 561-574.

16 van Rijt LS, Jung S, Kleinjan A, et al. In vivo depletion of lung $\mathrm{CD} 11 \mathrm{c}+$ dendritic cells during allergen challenge abrogates the characteristic features of asthma. J Exp Med 2005; 201: 981-991.

17 Nicolls MR, Taraseviciene-Stewart L, Rai PR, Badesch DB, Voelkel NF. Autoimmunity and pulmonary hypertension: a perspective. Eur Respir J 2005; 26: 1110-1118.

18 Tamby MC, Humbert M, Guilpain P, et al. Antibodies to fibroblasts in idiopathic and scleroderma-associated pulmonary hypertension. Eur Respir J 2006; 28: 799-807.

19 Morse JH, Barst RJ, Fotino M, Zhang Y, Flaster E, Fritzler MJ. Primary pulmonary hypertension: immunogenetic response to high-mobility group (HMG) proteins and histone. Clin Exp Immunol 1996; 106: 389-395.

20 van Rijt LS, Lambrecht BN. Dendritic cells in asthma: a function beyond sensitization. Clin Exp Allergy 2005; 35: 1125-1134.

21 Gabrilovich DI, Chen HL, Cunningham HT, et al. Production of vascular endothelial growth factor by human tumors inhibits the functional maturation of dendritic cells. Nat Med 1996; 2: 1096-1103.

22 Fainaru O, Woolf E, Lotem J, et al. Runx3 regulates mouse TGF-beta-mediated dendritic cell function and its absence results in airway inflammation. EMBO J 2004; 23: 969-979.

23 Kuipers H, Lambrecht BN. The interplay of dendritic cells, Th2 cells and regulatory $\mathrm{T}$ cells in asthma. Curr Opin Immunol 2004; 16: 702-708.

24 Steinman RM, Nussenzweig MC. Avoiding horror autotoxicus: the importance of dendritic cells in peripheral $\mathrm{T}$ cell tolerance. Proc Natl Acad Sci USA 2002; 99: 351-358.

25 Idzko $M$, Hammad $H$, Van Nimwegen $M$, Kool $M$, Hoogsteden HC, Lambrecht BN. Inhaled iloprost suppresses the cardinal features of asthma via inhibition of airway dendritic cell function. J Clin Invest 2007; (In press).

26 Zhou W, Hashimoto K, Goleniewska K, et al. Prostaglandin I2 analogs inhibit proinflammatory cytokine production and $\mathrm{T}$ cell stimulatory function of dendritic cells. J Immunol 2007; 178: 702-710.

27 Guruli G, Pflug BR, Pecher S, Makarenkova V, Shurin MR, Nelson JB. Function and survival of dendritic cells depend on endothelin-1 and endothelin receptor autocrine loops. Blood 2004; 104: 2107-2115.

28 Serafini P, Meckel K, Kelso M, et al. Phosphodiesterase-5 inhibition augments endogenous antitumor immunity by reducing myeloid-derived suppressor cell function. J Exp Med 2006; 203: 2691-2702.

29 Taieb J, Chaput N, Menard C, et al. A novel dendritic cell subset involved in tumor immunosurveillance. Nat Med 2006; 12: 214-219. 